\title{
The Increase in Protein Contour Length Depends on Mechanical Unfolding Conditions
}

\author{
A. DA̧Browska ${ }^{a, *}$, K. Lebed ${ }^{a}$, A.J. $\operatorname{KuliK}^{b}$, L. Forró $^{b}$ \\ AND M. LEKKA ${ }^{a}$ \\ ${ }^{a}$ The Henryk Niewodniczański Institute of Nuclear Physics \\ Polish Academy of Sciences \\ Radzikowskiego 152, 31-342 Kraków, Poland \\ ${ }^{b}$ Ecole Polytechnique Fédérale de Lausanne \\ Institute of Physics of Complex Matter, 1015 Lausanne, Switzerland \\ (Received July 20, 200\%; revised version December 5, 2007)
}

\begin{abstract}
Many proteins in alive organisms have a domain structure providing them the possibility to reversible unfolding, which seems to play an essential role in those processes occurring in tissues which are controlled by mechanical cellular tension. In this work the atomic force microscopy was applied to investigate the mechanical properties of the single molecules of fibronectin, a protein participating in the important mechanical processes in extracellular matrix. The results showed that the conditions of mechanical stretching influence not only the force required to unfolding of a domain but also the increase in protein contour length induced by such unfolding event. Two mean values of the increase in length (called shortly the unfolding length) $L_{1}$ and $L_{2}$, were obtained and ascribed to unfolding of either the whole fibronectin domain of type III $\left(L_{2}\right)$ or its fragment $\left(L_{1}\right)$. Both unfolding lengths revealed similar dependence on the stretching conditions. This experimental observation of increase in unfolding length with increasing loading rate was successfully described with a combination of two theoretical models (Bell model and the worm-like-chain model), previously used separately in the analysis of protein unfolding. The general mechanical property of fibronectin domains was emphasized and proposed as a potential determinant of the cellular adhesion.
\end{abstract}

PACS numbers: 87.14 Ee, 87.15.-v, 87.15.He, 87.15.La

\section{Introduction}

Many molecular processes are monitored by the activity of the protein binding sites. In modular proteins such binding sites may be hidden inside a folded do-

${ }^{*}$ corresponding author; e-mail: Aleksandra.Dabrowska@ifj.edu.pl 
main structure remaining inactive. The activation takes place as they get exposed outside during unfolding of a given domain. Moreover, the increase in protein contour length plays solely a crucial role in some biological processes. For example, in a giant muscle protein titin, a tandem of immunoglobulin- and FnIII-type domains is an essential source of passive elasticity of striated muscle [1-3].

Typically, modules (i.e. domains) are composed of $\beta$-strands structures and $\alpha$-helices which determines their mechanical stability $[4,5]$. Unfolding of a domain may occur as a result of action of chemical agent, temperature, or external force. The latter can be studied by means of atomic force microscopy (AFM). This technique enables stretching a single protein molecule and allows the measurement of the unfolding force (i.e. the force which induces the unfolding of a domain). The unfolding length (i.e. the mean value of the increases in contour length resulting from each released fragment of protein chain) can be obtained from the measurement as well.

In this work it was shown that the observed unfolding lengths corresponding to unfolding of a single domain depend on the conditions of mechanical stretching.

Fibronectin is an important component of the extracellular matrix. It plays an important mechanical role in tissues since it participates in cell adhesion and cell mobility $[6,7]$. The ability to unfolding of its domains was proved experimentally [8]. It is a long glycoprotein composed of two nearly identical monomers linked together by disulphide bridges near their carboxyl termini. Three types of domains can be distinguished in the tertiary structure of fibronectin. There are twelve domains of type I (FnI), two domains of type II (FnII) and about fifteen domains of type III (FnIII) [9]. The contour lengths of fibronectin domains of approximately $16 \mathrm{~nm}, 24 \mathrm{~nm}$, and $34 \mathrm{~nm}$ correspond to 44 (FnI), 63 (FnII), and 90 (FnIII) amino acids, respectively. Each domain has a $\beta$-strands structure maintained mainly by hydrogen bonds [5, 10].

The unfolding of a protein is a phenomenon in which, upon the stretching force, thermal fluctuations influence the unfolding events. Thus, the unfolding depends on how fast the unfolding process occurs, which was already studied for fibronectin [11-13]. However, to our knowledge, the influence of unfolding speed on the increase in protein contour length was not investigated experimentally.

In this work, the unfolding of fibronectin FnIII domains was analyzed with the special emphasis on the aspect how the mechanical parameters of stretching (retraction velocity and system spring constant) influence the increase in protein contour length.

\section{Materials and methods}

\subsection{Sample preparation}

Natural fibronectin from bovine plasma was purchased from Sigma. The fibronectin fragment FnIII ${ }^{4-12}$ was kindly provided by Prof. Harold P. Erickson (Dept. of Cell Biology, Duke University). Typical concentration of protein was 
$0.1 \mathrm{mg} / \mathrm{ml}$ (for natural $\mathrm{Fn}$ ) and $0.04 \mathrm{mg} / \mathrm{ml}$ (for FnIII ${ }^{4-12}$ ). In both cases, phosphate buffered saline (PBS, ICN Biomedicals, $\mathrm{pH} 7.4$, containing $10 \mathrm{mM}$ of $\mathrm{PO}_{4}^{2-}$, $137 \mathrm{mM}$ of $\mathrm{NaCl}$ and $27 \mathrm{mM}$ of $\mathrm{KCl}$ ) was used to prepare the protein solution.

Freshly cleaved mica, used as a substrate for fibronectin immobilization, was incubated in the protein solution for about 30 minutes. Then the sample was rinsed with PBS buffer and immediately measured by AFM.

\subsection{AFM measurements}

The measurements were carried out using two devices: a home-built AFM [14] and a commercial model CP (Park Scientific Instruments, Santa Barbara, USA). Standard silicon nitride cantilevers (MLCT - AUHW, from Veeco) with a nominal spring constants of $0.01 \mathrm{~N} / \mathrm{m}$ and $0.03 \mathrm{~N} / \mathrm{m}$ were used. No special treatment was applied to the cantilevers. The unfolding of fibronectin was performed in the liquid cell in the buffer environment. Force curves were acquired within the area of about $1 \mu \mathrm{m}^{2}$ at different locations on the sample surface. The step size was always less than $1 \mathrm{~nm}(0.3-0.97 \mathrm{~nm})$. The retraction velocity varied from 0.1 $\mu \mathrm{m} / \mathrm{s}$ to $1.6 \mu \mathrm{m} / \mathrm{s}$.

\section{Results and discussion}

\subsection{Mechanical unfolding of natural fibronectin}

Figure 1 presents the typical force curve recorded during stretching of a single molecule of natural fibronectin. It shows a characteristic saw-tooth pattern composed of several unfolding events.

The analysis of such data should follow criteria given by Best et al. [15]. According to them, an ideal unfolding curve contains regular peaks separated by a reproducible distance. The first and the last force drop should be excluded from the analysis, since the first one can be influenced by non-specific interactions and the last one usually represents the protein detachment from the tip. The analysis of the unfolding pattern provides two quantities: the value of increase in contour length, resulting from unraveling and stretching of a single domain, and the value of force needed to unfold such a single domain. The latter is defined as the maximum force value preceding a sharp drop [15] as it is shown in the inset of Fig. 1.

The increases in contour length, obtained in a single measurement, were used to create a histogram (Fig. 2). In each single measurement two peaks were reproducibly observed on the histograms of increases in length. Thus each two peaks were fitted with two Gauss functions which resulted in a pair of unfolding lengths, $L_{1}$ and $L_{2}$.

Fibronectin molecule is composed of three domain types of different contour length. All domains have $\beta$-sheet structure thus stabilized by hydrogen bonds. FnI and FnII domains, however, are additionally stabilized by strong internal disulphide bonds. This fact caused some doubts during the analysis of the AFM data obtained for fibronectin unfolding. In one of the first studies of the mechanical 


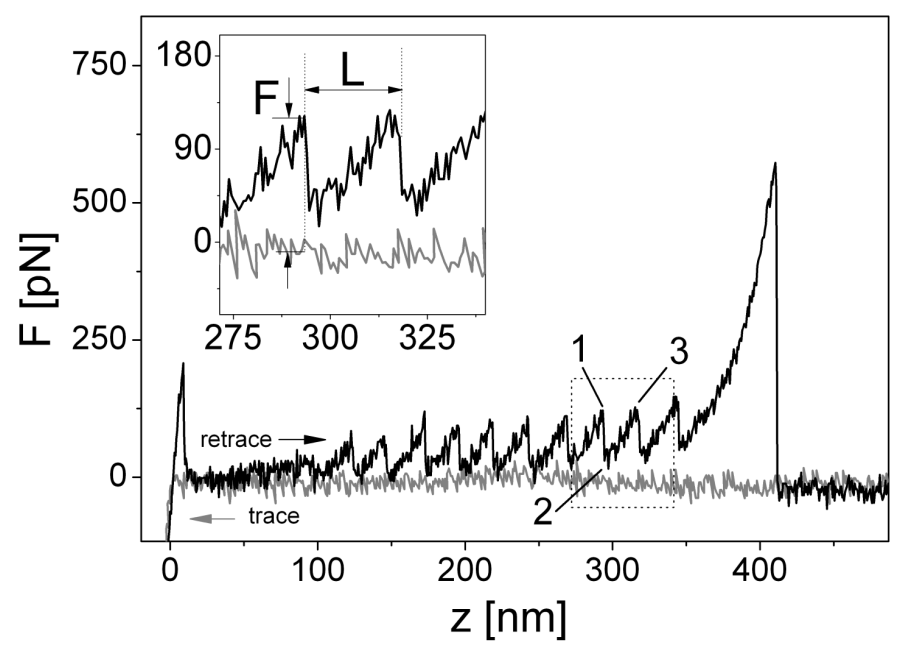

Fig. 1. A typical force curve showing multiple unfolding events resulting from a single fibronectin molecule stretching ( $z$ is end-to-end distance). Three characteristic steps of unfolding were marked by numbers: when the force reaches the critical value of unfolding force the unfolding of a protein takes place (1) causing a drop of force value (2) followed by increase in contour length (2-3). Inset: enlargement of the area marked by the dotted rectangle in the main figure shows the way of analysis of a single unfolding event. The unfolding force $F$ is defined as a maximum force value preceding a sharp drop. The unfolding length $L$ is a difference between subsequent peaks in the pattern.

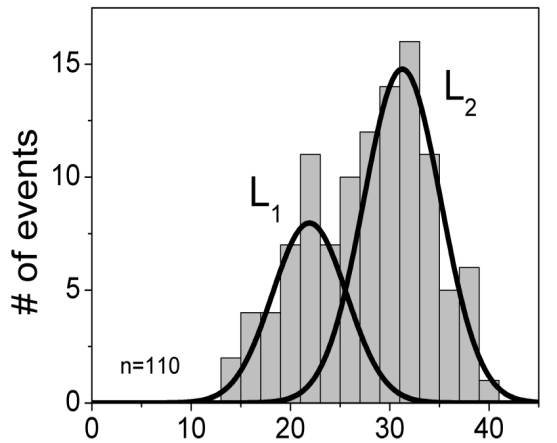

Fig. 2 L [nm]

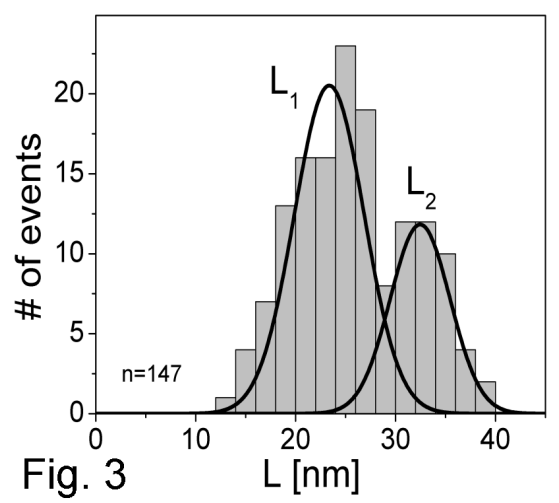

Fig. 2. The example of histogram of increases in contour length obtained for natural fibronectin. Two peaks were fitted with the Gauss functions, centered at $L_{1}=(21.3 \pm$ $3.7) \mathrm{nm}$ and $L_{2}=(31.3 \pm 3.9) \mathrm{nm}$, respectively (n denotes the number of unfolding events).

Fig. 3. The histogram of increases in contour length obtained for FnIII ${ }^{4-12}$ fibronectin fragment. The Gauss functions are centered at $L_{1}=(23.4 \pm 3.5) \mathrm{nm}$ and $L_{2}=(32.5 \pm 3.0)$ $\mathrm{nm}$, respectively ( $n$ denotes the number of unfolding events). 
stretching of natural fibronectin it was shown that all three domain types did unfold [11]. However, later Oberhauser et al. reported the lack of unfolding events corresponding to FnI and FnII domains and explained that fact by sufficiently strong stabilization of those structures by internal disulphide bonds [12].

The larger unfolding length $L_{2}$ of approximately $31 \mathrm{~nm}$ (corresponding to the second peak in the histogram in Fig. 2) can be related to unfolding of the entire FnIII domains since their mean contour length equals about $34 \mathrm{~nm}$. The smaller unfolding length $L_{1}$ of approximately $21 \mathrm{~nm}$ (corresponding to the first peak in the histogram in Fig. 2) is definitely larger than the mean contour length of FnI domains (about $16 \mathrm{~nm}$ ). Thus, the obtained value $L_{1}$ cannot result from FnI domains unfolding. It could be possible to ascribe $L_{1}$ to the unfolding of FnII domains for which the mean contour length equals approximately $24 \mathrm{~nm}$. However, there are only two such domains in one fibronectin monomer (four in a dimer), thus, concerning the ratio of numbers of FnII and FnIII domains in a single monomer (2:5), one could expect that FnII domains would always produce clearly smaller peak in the histogram which was not observed (see e.g. Fig. 3). It was concluded that unfolding length $L_{1}$ resulted from unfolding of domains of type III as well.

However, in order to unambiguously exclude the possibility that the smaller unfolding length $L_{1}$ is related in any other way with the presence of FnI and FnII domains, the analogous experiment was performed for fibronectin fragment composed of FnIII domains only (for recombinant FnIII ${ }^{4-12}$ fragment).

\subsection{Mechanical unfolding of FnIII ${ }^{4-12}$ fibronectin fragment}

In this case, for $\mathrm{FnIII}^{4-12}$ fragment, two separate unfolding lengths were similarly obtained. These reference values were found to be $L_{1}=(23.4 \pm 3.5)$ $\mathrm{nm}$ and $L_{2}=(32.5 \pm 3.0) \mathrm{nm}$, whereas the corresponding pair of values obtained previously for natural fibronectin was $L_{1}=(21.3 \pm 3.7) \mathrm{nm}$ and $L_{2}=(31.3 \pm 3.9)$ $\mathrm{nm}$

Therefore, each of two unfolding lengths occurring in both, natural and recombinant fibronectin molecules, can be attributed to unfolding of FnIII domains.

\subsection{Two values of the unfolding length}

It is possible that in one protein molecule some domains are already partially unfolded before the mechanical stretching. Therefore, there could be two populations of domains in one molecule, native (folded) and partially denatured. One of the reasons of obtaining the smaller unfolding length could be related to stretching of such partially denaturated structures. Another possibility is unfolding with an intermediate state. The two steps of unfolding result in increases in contour length which are smaller than the contour length of the whole domain.

In such cases, the unfolding of a part of domain should precede or follow the unfolding of the remaining folded part. The unfolding through intermediate state was analyzed in theoretical works concerning the unfolding of system composed of 
chosen fibronectin domains of type III, e.g. ${ }^{1} \mathrm{FnIII}[16],{ }^{9} \mathrm{FnIII}[17],{ }^{10} \mathrm{FnIII}[17$, 18]. Very well known secondary structure of studied domains allowed to ascribe the observed increases in length to unfolding of subsequent $\beta$-strands of a given domain. The results of simulations for ${ }^{9} \mathrm{FnIII}$ and ${ }^{10} \mathrm{FnIII}$ done by Paci and Karplus [17] provided a few possible intermediate states: at protein extension of about $I_{1}=5.5 \mathrm{~nm}, I_{2}=15 \mathrm{~nm}$ and $I_{\max }=32 \mathrm{~nm}$. Later numerical simulations done by Gao et al. [18] for ${ }^{10} \mathrm{FnIII}$ domain confirmed the presence of all these intermediate states $\left(I_{1}=5.6 \mathrm{~nm}, I_{2}=13.5 \mathrm{~nm}\right.$ and $\left.I_{\max }=31.5 \mathrm{~nm}\right) . I_{1}$ and $I_{2}$ were ascribed to the breaking of hydrogen bonds binding the subsequent pairs of $\beta$-strands in fibronectin domain. The intermediate state $I_{1}$ corresponds to the breaking bonds between three $\beta$-strands which enables the unfolding of first two terminal $\beta$-strands of a domain while $I_{2}$ corresponds to the breaking of all bonds in a domain besides those between last two bonded strands [16]. $I_{\max }$ corresponds obviously to the fully stretched domain.

Next, Gao et al. [16] reported the intermediate states at similar extensions of $I_{1}=5.5 \mathrm{~nm}, I_{2}=15 \mathrm{~nm}$ and $I_{\max }=33 \mathrm{~nm}$ as a results of simulation performed for ${ }^{1}$ FnIII domain. All FnIII domains mentioned above have very similar secondary structure thus all obtained intermediate states result from the unfolding of the same pairs of $\beta$-strands. A part of experimental results are in good agreement with theoretical ones. Oberhauser et al. [12] reported two experimentally obtained unfolding lengths for $1 \mathrm{FnIII}$ (about $9 \mathrm{~nm}$ and $20 \mathrm{~nm}$ ) and also similar values were obtained by Li et al. [19] for ${ }^{10} \mathrm{FnIII}$ (about $12 \mathrm{~nm}$ and $19 \mathrm{~nm}$ ). Those two pairs of experimental values of unfolding lengths can be compared with results of simulations [16-18]. The difference between two positions of intermediate states, $I_{\max }$ and $I_{2}$, corresponds to the larger increases in length obtained from the experiment (i.e. $20 \mathrm{~nm}$ [12] or $19 \mathrm{~nm}$ [19]). In turn, the smaller increases in length (i.e. 9 $\mathrm{nm}$ [12] or $12 \mathrm{~nm}$ [19]) can be compared with the position of intermediate state $I_{2}$ reduced by the size of a folded domain, thus approximately of $3.5 \mathrm{~nm}$ [20].

Native protein consists of many different kinds of domains. However, the experiment performed by Abu-Lail et al. [21] provided the important evidence that partial unfolding events are possible to observe effectively for the system composed of different domains of type III. For the FnIII ${ }^{1-8}$ fibronectin fragment they observed three unfolding lengths of about $10.6 \mathrm{~nm}, 20 \mathrm{~nm}$, and $28.7 \mathrm{~nm}$. First two values were already discussed above and the last one corresponds obviously to unfolding of the entire domain without any intermediate state.

In order to ascribe the unfolding length $L_{1}$ to a given domain fragment, it seemed to be more appropriate to analyze the contour length $L_{\mathrm{C} 1}$ of unknown domain fragment, provided by Eq. (3.3) (see Fig. 4B, Sect. 3.4) applied for the set of obtained values $L_{1}$.

The obtained contour length $L_{\mathrm{C} 1}=(27 \pm 1.5) \mathrm{nm}$ value reduced by the average size of a folded domain is larger than the experimental values obtained 

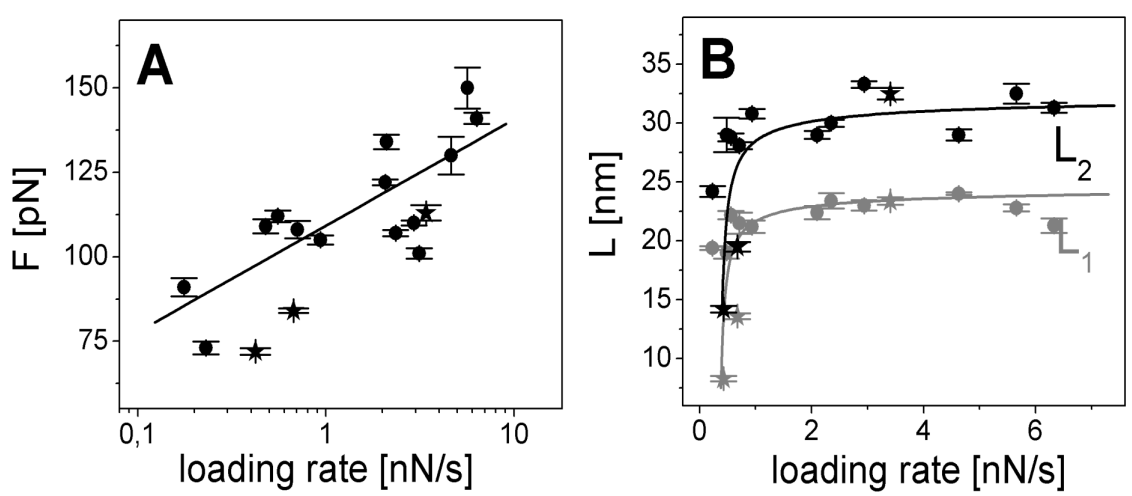

Fig. 4. The influence of loading rate on fibronectin unfolding. (A) The unfolding force as a function of loading rate. Bell model parameters calculated from the slope and the intercept of the linear function are equal to $r_{0}=0.30 \mathrm{~nm}$ and $k_{0}=0.023 \mathrm{~s}^{-1}$. (B) The relation between unfolding lengths and loading rate. Both lengths, $L_{1}$ and $L_{2}$, show similar dependence on loading rates. Black and gray solid curves were obtained from Eq. (3.3) with $r_{0}$ and $k_{0}$ values given above and $p=0.45 \mathrm{~nm}$ assumed for the WLC model. The contour lengths obtained from Eq. (3.3) equal $L_{\mathrm{C} 1}=(27 \pm 1.5) \mathrm{nm}$ for the fragment and $L_{\mathrm{C} 1}=(35.5 \pm 2.5) \mathrm{nm}$ for the whole domain. The error bars in (A) and (B) represent the standard deviation of the mean. The reference points obtained for FnIII $^{4-12}$ are marked with stars.

previously and discussed above. Thus, the unfolding length $L_{\mathrm{C} 1}$ cannot result form unfolding through the considered intermediate state.

Additionally, no unfolding of the rest domain characterized by a contour length of about $8.5 \mathrm{~nm}\left(L_{\mathrm{C} 2}-L_{\mathrm{C} 1}\right)$, neither preceding nor following the increase $L_{\mathrm{C} 1}$, was observed. Therefore, the only explanation for obtaining of LC1 length is the unfolding of the partially denatured domains.

The lacking denatured fragments of about $8.5 \mathrm{~nm}$ corresponding to about $22 \pm 5$ amino acids could be assigned to one of two pairs of terminal $\beta$-strands in FnIII domain, namely $\mathrm{A}$ and $\mathrm{B}$ or $\mathrm{F}$ and $\mathrm{G}$ (compare with $[16,18]$ ).

\subsection{The influence of measurement conditions on the unfolding force and the increase in contour length}

The unfolding of natural fibronectin was studied as a function of a loading rate, a parameter describing how fast the external force applied to the protein molecule increases in time during unfolding. The loading rate can be estimated by multiplying the retraction velocity by the system spring constant (taking into account both, the cantilever spring constant and the protein stiffness - two springs linked in series).

Bell model [22], initially created for description of the rupture of specific interactions in the complex of ligand - receptor, was then successfully used to describe the unfolding of modular proteins [23]. This model predicts how free 
energy of the system changes with the external force in the direction of stretching. The transition from folded to unfolded state, involves overcoming the potential barrier which occurs under applied force on a distance $r_{0}$. In other words, the distance $r_{0}$ (called also the potential width of unfolding) is the distance between folded state (i.e. potential minimum) and the transition state (i.e. the maximum of the potential) [24].

According to the Bell model, the relation between the mean unfolding force $f_{\text {crit }}$ and logarithm of the loading rate $r_{f}$ should be linear. The obtained results are presented in Fig. 4A. The linear region was observed and fitted with the line using the relation provided by Bell model

$$
f_{\text {crit }}\left(r_{f}\right)=\frac{k_{\mathrm{B}} T}{r_{0}} \ln \left(\frac{r_{0} r_{f}}{k_{0} k_{B} T}\right),
$$

where $r_{f}$ is the loading rate, $r_{0}$ - the potential width of unfolding, $K_{0}$ - the unfolding rate at zero force, $T$ - the absolute temperature and $k_{\mathrm{B}}$ - the Boltzmann constant. Based on this model, $r_{0}$ and $k_{0}$ parameters were calculated from the slope and the intercept of the linear range, respectively. The values $r_{0}=0.30$ $\mathrm{nm}$ and $k_{0}=0.023 \mathrm{~s}^{-1}$ obtained as average values for 15 domains $\left(\mathrm{FnIII}^{1-15}\right)$ are comparable with the results reported by Oberhauser et al.: $r_{0}=\left\{0.17 \mathrm{~nm}\left(\mathrm{Fn}^{1,2}\right)\right.$ $\left.-0.38 \mathrm{~nm}\left(\mathrm{Fn}^{10}\right)\right\}$ and $k_{0}=\left\{0.004 \mathrm{~s}^{-1}\left(\mathrm{Fn}^{1,2}\right)-0.022 \mathrm{~s}^{-1}\left(\mathrm{Fn}^{13}\right)\right\}[10]$.

Subsequently, the influence of the loading rate on the increase in length was investigated. It turned out that the unfolding lengths observed in our experiment reveal clear increase with the increase in loading rate (Fig. 4B). In studied range of loading rates, the change in values of unfolding lengths was about $100 \%$ for $L_{1}$ and $80 \%$ for $L_{2}$. These experimental results were described using both, Bell model and the worm-like-chain (WLC) model [25]. WLC model describes a protein chain deformation under the external force. The force $f$ required to extend the protein chain by a given length $z$ is the following:

$$
f(z)=\frac{k_{\mathrm{B}} T}{p}\left[\frac{1}{4\left(1-z / L_{0}\right)^{2}}-\frac{1}{4}+\frac{z}{L_{0}}\right],
$$

where $p$ is the persistence length, $L_{0}$ - the contour length of the studied domain fragment, $T$ - the absolute temperature and $k_{\mathrm{B}}$ - the Boltzmann constant.

Bell model gives the most probable force (at a given loading rate) which can be achieved during stretching the $i$-th unfolded domain, before the next, $(i+1)$-th domain will unfold. Substitution the critical force value from Eq. (3.1) to Eq. (3.2) allows calculating of the loading rate value necessary to extend the protein domain to the required length $z$ :

$$
r_{f}(z)=\frac{k_{0} k_{\mathrm{B}} T}{r_{0}} \exp \left[\frac{r_{0}}{p}\left(\frac{1}{4\left(1-z / L_{0}\right)^{2}}-\frac{1}{4}+\frac{z}{L_{0}}\right)\right]
$$

The fitting of obtained unfolding patterns was succeeded using a persistence length of $0.45 \mathrm{~nm}$, the value comparable with those obtained previously [26, 27]. This $p$ value and $r_{0}$ and $k_{0}$ values obtained above were used to plot the function described with combination of Bell and WLC models (Fig. 4B). As can be seen from 
the comparison of experimental data with the plots, the relation (3.3) reproduces the dependence of both unfolding lengths, $L_{1}$ and $L_{2}$ on the loading rate. Higher loading rates enable to achieve larger forces during stretching of a molecule. The latter enable the larger extensions of the released protein fragments. With increasing loading rate the unfolding length value achieves the saturation level determined by the contour length of the domain $\left(L_{\mathrm{C} 2}\right)$ or its fragment $\left(L_{\mathrm{C} 1}\right)$.

\section{Summary}

Elasticity of tissues is believed to be related to elastic properties of proteins like collagen and fibronectin. As an important component of extracellular matrix fibronectin plays an essential role in cellular adhesion. Those two functions are related to two properties of this protein: ability to reversible unfolding under applied tension and the ability to specific interaction (with integrin; reviewed in [28]). Assuming that the rate of increase in extracellular tension can be described with the value of loading rate parameter one can expect that the obtained results will reflect the mechanical reaction of fibronectin molecules in tissue. The observed dependence of unfolding lengths on the rate of applied tension may therefore shed the light on the way of controlling the preservation of the adhesion contacts under elastic reorganization of the tissue.

Similar behavior was observed for fully unfolded domains as well as for domains partially denaturated. Thus, the general property of modular motifs under mechanical tension can be concluded.

\section{Acknowledgments}

The authors thank to Prof. Harold P. Erickson (Department of Cell Biology, Duke University, USA) for providing the fibronectin fragment NNIII $^{4-12}$ and to Prof. Piotr Marszaek (Department of Mechanical Engineering and Materials Science, Duke University, USA) for valuable remarks.

\section{References}

[1] M. Rief, M. Gautel, F. Oesterhelt, J.M. Fernandez, H.E. Gaub, Science 276, 1109 (1997).

[2] W.A. Linke, D.E. Rudy, T. Centner, M. Gautel, Ch. Witt, S. Labeit, C.C. Gregorio, J. Cell Biol. 146, 631 (1999).

[3] A. Minajeva, M. Kulke, J.M. Fernandez, W.A. Linke, Biophys. J. 80, 1442 (2001).

[4] M. Carrion-Vazquez, A. Oberhauser, T. Fisher, P. Marszalek, H. Li, J. Fernandez, Prog. Biophys. Mol. Biol. 74, 63 (2000).

[5] H. Lu, K. Schulten, Proteins 35, 453 (1999).

[6] K.M. Yamada. J. Clin. Inves. 105, 1507 (2000).

[7] R.O. Hynes, Proc. Natl. Acad. Sci. USA 96, 2588 (1999).

[8] T. Ohashi, D.P. Kiehart, H.P. Erickson, Proc. Natl. Acad. Sci. USA 96, 2153 (1999). 
[9] J.R. Potts, I.D. Campbell, Curr. Opin. Cell Biol. 6, 648 (1994).

[10] A. Krammer, H. Lu, B. Isralewitz, K. Schulten, V. Vogel, Proc. Natl. Acad. Sci. USA 96, 1351 (1999).

[11] Y. Oberdrfer, H. Fuchs, A, Janshoff, Langmuir 16, 9955 (2000).

[12] A.F. Oberhauser, C. Badilla-Fernandez, M. Carrion- Vazquez, J.M. Fernandez, J. Mol. Biol. 319, 433 (2002).

[13] P.Y. Meadows, J.E. Bemis, G.C. Walker, Langmuir 19, 9566 (2003).

[14] M. Lekka, J. Lekki, M. Marszałek, P. Golonka, Z. Stachura, B. Cleff, A. Hrynkiewicz, Appl. Surf. Sci. 141, 345 (1999).

[15] R.B. Best, D.J. Brockwell, J.L. Toca-Herrera, A.W. Blake, D.A. Smith, S.E Radford, J. Clarke, Anal. Chim. Acta 479, 87 (2002).

[16] M. Gao, D. Craig, O. Lequin, I.D. Campbell, V. Vogel, K. Schulten, Proc. Natl. Acad. Sci. USA 100, 14784 (2003).

[17] E. Paci, M. Karplus J. Mol. Biol. 288, 441 (1999).

[18] M. Gao, D.W. Craig, V. Vogel, K. Schulten, J. Mol. Biol. 323, 939 (2002).

[19] L. Li, H. Han-Li Huang, C. L. Badilla, J. M. Fernandez, J. Mol. Biol. 345, 817 (2005).

[20] H.P. Erickson, Proc. Natl. Acad. Sci. USA 91, 10114 (1994).

[21] N.I. Abu-Lail, T. Ohashi, R.L. Clark, H.P. Erickson, S. Zauscher, Matrix Biol. 25, 175 (2006).

[22] E. Evans, K. Ritchie, Biophys. J. 72, 1541 (1997).

[23] M. Rief, M. Gautel, F. Oesterhelt, J.M. Fernadez, H.E. Gaub, Science 276, 1109 (1997).

[24] R. Best, S. Fowler, J. Toca-Herrera, J. Clarke, Proc. Natl. Acad. Sci. USA 99, 12143 (2001).

[25] J.F. Marko, E.D. Siggia, Macromolecules 28, 8759 (1995).

[26] A.F. Oberhauser, P.E. Marszaek, H.P. Erickson, J.M. Fernandez, Nature 393, 181 (1998).

[27] M. Rief, M. Gautel, A. Schemmel, H.E. Gaub, Biophys. J. 75, 3008 (1998).

[28] S. Johansson, G. Svineng, K. Wennerberg, A. Armulik, L. Lohikangas, Front. Biosci. 2, 126 (1997). 